\title{
EXOTIC HIGGS DECAYS IN U(1) EXTENSIONS OF THE MSSM
}

\author{
Peter Athron \\ ARC Centre of Excellence for Particle Physics at the Terascale, School of Physics, \\ Monash University, Melbourne, Victoria 3800, Australia \\ Margarete Mühlleitner \\ Institute for Theoretical Physics, Karlsruhe Institute of Technology, 76128 \\ Karlsruhe, Germany \\ Roman Nevzorov and Anthony G. Williams \\ ARC Centre of Excellence for Particle Physics at the Terascale and CSSM, School \\ of Chemistry and Physics, University of Adelaide, Adelaide SA 5005, Australia \\ Abstract. We study the decays of the lightest $\mathrm{CP}-$ even Higgs boson into a pair of \\ pseudoscalar Higgs states within $U(1)_{N}$ extensions of the MSSM.
}

\section{Introduction}

A new bosonic state with a mass around $125 \mathrm{GeV}$, which was discovered at the LHC a few years ago, can provide a window into new physics beyond the Standard Model (SM). The signal strengths associated with this particle are currently consistent with the SM Higgs boson. However further exploration of this resonance can lead to new exciting discoveries. Physics beyond the SM may change the Higgs decay rates to SM particles and give rise to new decay modes. Here we consider the decays of the SM-like Higgs boson into a pair of pseudoscalar states within $U(1)$ extensions of the Minimal Supersymmetric Standard Model (MSSM). In particular we focus on the $E_{6}$ inspired SUSY models which are based on the low-energy SM gauge group together with an extra $U(1)_{N}$ gauge symmetry defined by:

$$
U(1)_{N}=\frac{1}{4} U(1)_{\chi}+\frac{\sqrt{15}}{4} U(1)_{\psi} .
$$

Gauge $U(1)_{\psi}$ and $U(1)_{\chi}$ symmetries can originate from the breakings $E_{6} \rightarrow$ $S O(10) \times U(1)_{\psi}, S O(10) \rightarrow S U(5) \times U(1)_{\chi}$. Different aspects of the phenomenology of these $E_{6}$ inspired supersymmetric (SUSY) models have been extensively studied in $[1,2,3,4,5,6,7,8,9,10,11,12,13$.

\section{The $U(1)_{N}$ extensions of the MSSM}

These SUSY models imply that just below the scale $M_{X}$ the gauge symmetry in the $E_{6}$ Grand Unified Theory (GUT) is broken down to $S U(3)_{C} \times S U(2)_{W} \times$ $U(1)_{Y} \times U(1)_{N} \times Z_{2}^{M}$, where $Z_{2}^{M}=(-1)^{3(B-L)}$ is a matter parity. To ensure anomaly cancellation, gauge coupling unification and the appropriate breakdown of the gauge symmetry at low energies the matter content of the SUSY 
models under consideration involves three 27 representations of the $E_{6}$, two $S U(2)_{W}$ doublets $L_{4}$ and $\bar{L}_{4}$ from extra $27_{1}^{\prime}$ and $\overline{27^{\prime}}{ }_{1}$, two SM singlets $S$ and $\bar{S}$ that carry non-zero $U(1)_{N}$ charges and stem from another pair of $27_{2}^{\prime}$ and $\overline{27}_{2}$ as well as a SM singlet $\phi$ that does not participate in gauge interactions. Thus these models involve extra matter beyond the MSSM. In particular, they include three pairs of $S U(2)_{W}$-doublets $\left(H_{i}^{d}\right.$ and $\left.H_{i}^{u}\right)$ that have the quantum numbers of Higgs doublets, and three pairs of colour triplets of exotic quarks $\bar{D}_{i}$ and $D_{i}$ with electric charges $+1 / 3$ and $-1 / 3$ respectively. To suppress flavour changing neutral currents as well as the most dangerous baryon and lepton number violating operators one can impose a discrete $\tilde{Z}_{2}^{H}$ symmetry, under which all superfields except one pair of $H_{i}^{d}$ and $H_{i}^{u}$ (say $H_{d} \equiv H_{3}^{d}$ and $\left.H_{u} \equiv H_{3}^{u}\right), L_{4}, \bar{L}_{4}, S, \bar{S}$ and $\phi$ are odd.

The conservation of $Z_{2}^{M}$ and $\tilde{Z}_{2}^{H}$ symmetries implies that these models contain at least two states which are absolutely stable and can contribute to the dark matter density. One of these states is a lightest SUSY particle (LSP) whereas another one is the lightest ordinary neutralino. Using the method proposed in [14] it was argued that there is a theoretical upper bound on the mass of the LSP [7]. In the simplest phenomenologically viable scenarios the LSP has a mass around $1 \mathrm{eV}$ or even smaller forming hot dark matter in the Universe. The presence of such very light neutral fermions in the particle spectrum may have interesting implications for neutrino physics (see, for example [15]). In these scenarios the lightest ordinary neutralino can account for all or some of the observed dark matter density.

\section{Non-Standard Higgs Decays}

The Higgs sector of the SUSY models under consideration contains two Higgs doublets $H_{d}$ and $H_{u}$ as well as $S, \bar{S}$ and $\phi$. The scalar components of the superfields $S$ and $\bar{S}$ acquire large vacuum expectation values (VEVs) that break the $U(1)_{N}$ gauge symmetry inducing the masses of exotic particles and the $Z^{\prime}$ boson. The interaction of $S, \bar{S}$ and $\phi$ is determined by the following terms in the superpotential that can be written as

$$
W_{S}=\sigma \phi S \bar{S}+\frac{\kappa}{3} \phi^{3}+\frac{\mu}{2} \phi^{2}+\Lambda \phi .
$$

When the couplings $\kappa, \mu$ and $\Lambda$ are rather small these models possess an approximate global $U(1)$ symmetry. This symmetry is spontaneously broken by the VEVs of $S, \bar{S}$ and $\phi$ leading to the presence of a light pseudoscalar state in the particle spectrum. Our analysis indicates that the couplings of this state to the SM particles are always small. Therefore even if this pseudoscalar is substantially lighter than $100 \mathrm{GeV}$ it could escape detection at former and present collider experiments. Moreover this state can be so light that it gives rise to the decays of the SM-like Higgs boson into a pair of these pseudoscalar states. 
The branching ratio of such decays depends rather strongly on the values of the couplings that explicitly violate the global $U(1)$ symmetry.

To simplify our analysis we set $\mu=0$ as well as $\Lambda=0$ and examine the dependence of the branching ratio of the exotic Higgs decays on $\kappa$. For $\kappa \sim$ 0.001 the pseudoscalar Higgs boson with a mass of $40-60 \mathrm{GeV}$ can be obtained without fine-tuning but the branching ratio of the SM-like Higgs decays into a pair of such particles is smaller than $10^{-4} 13$. Thus it will be rather difficult to observe such decays at the LHC. If $\kappa>0.01$ a fine tuning of at least $1 \%$ is required to get the lightest CP-odd Higgs state with mass of $40-60 \mathrm{GeV}$. However the branching ratio of the corresponding exotic Higgs decays can be larger than $1 \%$ in this case [13]. After being produced from such decays the lightest pseudoscalars decay into a pair of $b$-quarks or $\tau$-leptons resulting in four fermion final states.

\section{Acknowledgments}

This work was supported by the University of Adelaide and the Australian Research Council through the ARC Center of Excellence in Particle Physics at the Terascale.

[1] S. F. King, S. Moretti, R. Nevzorov, Phys. Rev. D 73, 035009 (2006).

[2] S. F. King, S. Moretti, R. Nevzorov, Phys. Lett. B 634, 278 (2006).

[3] S. F. King, S. Moretti, R. Nevzorov, Phys. Lett. B 650, 57 (2007).

[4] S. F. King, R. Luo, D. J. Miller, R. Nevzorov, JHEP 0812, 042 (2008).

[5] P. Athron, S. F. King, D. J. Miller, S. Moretti, R. Nevzorov, Phys. Lett. B 681, 448 (2009).

[6] P. Athron, S. F. King, D. J. Miller, S. Moretti, R. Nevzorov, Phys. Rev. D 80, 035009 (2009).

[7] J. P. Hall, S. F. King, R. Nevzorov, S. Pakvasa, M. Sher, Phys. Rev. D 83, 075013 (2011).

[8] P. Athron, S. F. King, D. J. Miller, S. Moretti, R. Nevzorov, Phys. Rev. D 84, 055006 (2011).

[9] R. Nevzorov, Phys. Rev. D 87, 015029 (2013).

[10] P. Athron, S. F. King, D. J. Miller, S. Moretti, R. Nevzorov, Phys. Rev. D 86, 095003 (2012).

[11] R. Nevzorov, S. Pakvasa, Phys. Lett. B 728, 210 (2014).

[12] R. Nevzorov, Phys. Rev. D 89, 055010 (2014).

[13] P. Athron, M. Mühlleitner, R. Nevzorov, A. G. Williams, JHEP 1501, $153(2015)$.

[14] S. Hesselbach, D. J. Miller, G. Moortgat-Pick, R. Nevzorov, M. Trusov, Phys. Lett. B 662, 199 (2008).

[15] J. M. Frere, R. Nevzorov, M. I. Vysotsky, Phys. Lett. B 394, 127 (1997). 